\title{
Research of Medical and Nursing Integration Model based on Family Doctor
}

\author{
Juning Wang \\ School of Medicine, Xi'an Peihua University, Xi'an, Shaanxi, 710125
}

Keywords: Medical Doctor, Family Doctor, Medical combination

\begin{abstract}
This paper briefly introduces the concept of "medical and nursing integration" and summarizes the three forms of "medical and nursing integration". It is emphasized that the current development of the "medical and nursing integration" model should focus on the form of contracted family doctors, and explain from the perspective of the needs of the elderly, the economy and traditional cultural ethics, and reasons for the contracted family doctor form being the best form of "medical and nursing integration".
\end{abstract}

\section{Introduction}

In the world, China is one of the most populous countries, facing an increasingly aging population. How to support the elderly has become a key issue in the society. As far as China's aged care services are concerned, medical services are mostly separate models. The combination of medical and nursing mode came into being. The combination of medical care and maintenance is an opportunity for the transformation of old-age care institutions. With the aging of the aging, the traditional old-age care institutions only have the single function of "nurturing" in the face of the elderly service groups with a large population base. In the case of relatively scarce medical resources, the elderly who are sick are "helpful", and the shortcomings and shortcomings of traditional old-age care institutions can lead to bottlenecks in their own development. The new model of "healing and health care" can greatly alleviate the pressure of old-age care in the society. The medical services of the elderly extend from traditional hospitals and family beds to old-age institutions, and the fields expand to the periphery to alleviate the pressure on professional institutions. In this mode, community nurses play an important role as family doctor assistants. As follows, I will combine my work experience to summarize the role of family doctor assistants in the combination model.

\section{The role of family doctors in the combination of medical care}

For most elderly patients with family beds, they are basically accompanied by chronic diseases, and family doctor assistants not only have relevant experience in aged care, but also have more comprehensive community nursing expertise and knowledge, so they can provide relatively professional in nursing. service. I randomly selected the clinical data of 325 family bed patients in this community service center from December 2015 to December 2016. The study found that 93.1\% of family bed patients were over 60 years old and above 80 years old. Elderly patients have a relatively high probability of establishing a bed, up to $49.36 \%$. It can be seen that for elderly patients, there is a relatively large demand for family beds, and with the growing age of patients, in terms of family beds, it also has a growing market share, so it can be inferred that the elderly Patients are more likely to rely on family doctor assistants. It can be seen that the best choice for elderly patients to carry out medical rehabilitation services is the combination of medical and nursing modes. The survey also found that the highest proportion of all patients were cerebrovascular accident patients, the proportion was as high as $72.1 \%$, accounting for the second highest proportion of patients with chronic heart failure, the proportion was $12.13 \%$, followed by fractures, chronic lungs, etc. Diseases, it can be seen that chronic diseases are an important factor in the burden of old age. In the process of family bed service, the family doctor assistant has a more professional continuation of chronic disease care ability, and also has a strong health professional 
skills for elderly patients. Therefore, when nursing elderly patients, they can play more effective medical services.

As far as family doctor-style services are concerned, the core of the community is the health power of the community, and its main management content is the physical health of the residents. Based on the basic principles of full notification, voluntary contracting, freedom of choice, and service norms, the community family doctors team and needs The serviced family signs the agreement to provide residents with a healthy and continuous and integrated service in a timely manner. This type of service model has its own advantages. After establishing a service relationship with an elderly patient family, it provides patients with dynamic health management and medical services. Under this service model, there are mainly general practitioners and community nurses, while the latter It is also the assistant of the former, mainly providing active home-based professional services for bedridden patients and elderly patients such as chronic diseases, providing reasonable health education for patients, and providing professional services for their medication, rehabilitation, physical examination, and prevention of pressure sores. Medical care. The survey found that home management has a wide range of content, and family doctor assistants also carried out $47.3 \%$ of basic medical care, $14.1 \%$ of rehabilitation care and $38.6 \%$ of home care guidance for patients, and timely met the medical care of elderly patients. Demand plays an important role in the mode of combining health care.

\section{Signing family doctor form is the best "medical combination" mode}

Most older people have more or less health problems and need stable and convenient medical services. The first two forms of "health-care combination" oriented by the inter-institutional cooperation between the old-age care institutions and the medical institutions can enable the elderly to obtain regular visits, rehabilitation and treatment, and the opening of cooperative hospitals when they are ill. The Green Channel is getting medical services more quickly. The third type of contracted family doctor can provide a variety of services for the elderly in the community. First of all, for elderly people with relatively good physical condition and convenient travel, they can see and take medicines in medical service organizations in the community. In particular, many patients with chronic diseases such as heart disease, diabetes, and high blood pressure who need long-term medication can extend the single-dispensing amount in many local community medical institutions to facilitate patients. Second, for elderly people who need treatment and can be treated at home, such as infusion therapy, contracted doctors can visit and provide services according to their needs, eliminating the need for elderly patients, especially elderly patients with limited mobility, to make unnecessary movements. You can get treatment at home. Third, for elderly patients who need rehabilitation services, family doctors can provide medical services to help patients who are still in recovery after discharge. They can also tell their families how to provide reasonable medication, care and rehabilitation for elderly patients. Fourth, for elderly patients who need to receive soothing care, such as elderly people who need hospice care, family doctors can help the elderly to spend the last period of their lives at home. At the same time as the family doctor conducts routine basic medical treatment, it can also provide the elderly family members with the nursing guidance needed for hospice care, helping the elderly to better pass the dying state. For elderly patients who need to receive soothing treatment, such as patients who need supportive care, family doctors can also go to the stomach to replace the stomach tube, catheter. At present, the " $1+1+1$ " combination signing model advocated by the government means that an elderly person signing a family doctor and his team at the same time, and signing a second-level hospital and a third-level hospital to provide certain treatment for the elderly. Degree of freedom. By signing up for the family doctor service model and using the community medical service resources, it is more convenient for elderly people living at home to see a doctor. The elderly save the boat trips to the relatively distant second- and third-tier hospitals, and there is no need to queue up in the clinic for a long time. For many elderly people who need to take care of their families, there is no need to exhaust the physical and mental energy of the elderly and their family caregivers due to frequent visits to the hospital. Especially for the latter three elderly people who need rehabilitation services or soothing care, the family doctor 
service model allows them to support their families in the family without having to separate them from their families because of the need for these services.

At present, the old-age institutions and the surrounding medical institutions agree to cooperate or the two self-organized medical institutions within the old-age care institutions provide the medical services for the elderly. The two types of "medical and nursing” forms require relatively high economic capacity for the elderly or elderly families. For example, in 2013, the first "medical and nursing" pilot medical care institution established by the Beijing Municipal Government, in addition to basic medical treatment, as well as a number of clinics such as rehabilitation medicine and internal surgery, it is very convenient to see a doctor. However, the monthly rent for this nursing home is a minimum charge of 11,000 yuan for the self-care for the elderly. The monthly fee for the elderly is at least 12,650 yuan, which is very expensive compared to the previous. In many other "health-care" medical care institutions, the universal fees are also very high, especially when compared with home-based pensions.

Whether it is the combination of the first two types of old-age care institutions and medical institutions, or the form of "medical combination" that is approved to open medical institutions, or the form of contracted family doctors, in the case of better implementation, it conforms to the traditional spirit of "treatment without treatment". "Immediate treatment is not diseased" emphasizes that the elderly who are caring for the elderly in the organization, if there is a doctor to visit the clinic regularly, inform the health aspects of the precautions, to obtain the current disease and to improve according to the doctor's advice, etc. To help the elderly prevent problems in a certain degree, avoid some health problems or diseases caused by lack of doctors' medical health guidelines. The form of signing a family doctor can better help the elderly at home to prevent their health as much as possible. In this form, the family doctors and their teams signed by the elderly need to go on a regular basis (the requirements vary from place to place, but usually 1 or 2 times a week). The relevant indicators are measured. If the indicators are too high or too low, they will be carried out. Further examination. In the absence of regular monitoring by family doctors, the elderly generally cannot go to the hospital for frequent index examinations. Instead, they will go to the hospital when they are not feeling well, and may miss the opportunity for better treatment. At the same time, family doctors provide comprehensive health guidance for the elderly and their family members, including diet, nursing, medication, exercise, etc., to prevent physical discomfort or illness caused by bad habits as much as possible.

Under the current circumstances, existing community medical institutions should increase the promotion of family doctors' signing models by means of health talks, community clinics, setting up publicity boards and distributing brochures. The government should also advertise on television or radio to promote the family doctor's propaganda model. Let community residents, especially the elderly, know the existence of the family doctor contract model and its service content. Older people and other community residents will gradually change their concept of treatment in the process after they realize the actual benefits brought by the contracted family doctor. At the same time, the government should promote grassroots medical institutions to establish an information platform. First, through the community doctors to establish a patient's electronic health record, record the patient's comprehensive health information and medical history, and network with the higher-level medical institutions, to facilitate the referral of patients in the hospital after the second

and third-level hospitals. If the patient changes to a family doctor, it is also convenient for follow-up doctors to understand their health. The second is to establish an information platform for family doctors and patients (for the elderly may be family members) to communicate with each other. In addition to the phone, non-emergency patients or family members of the patient can interact with the doctor through the information platform to help patients understand their situation, obtain health information, and so on.

\section{Conclusion}

In the mode of medical integration, the majority of community nurses play an important role as family doctor assistants. Therefore, we should seize the opportunity to fully exert our professional 
influence and use our own nursing advantages for more family bed patients and chronic diseases. Residents provide a wide range of nursing services, allowing them to contribute to the development of a better combination of health care.

\section{Acknowledgements}

Fund Project: Fund Project: Fund Project Shaanxi Xi'an Social Science Planning Fund Project in 2017 Project No. 17PH18

\section{References}

[1] Wang Weimin. Community nurses combined with nursing activities to carry out health education [J]. Chinese Medicine Guide, 2014 (01): 37-38.

[2] Wu Zhenyun, Li Juan, Xu Shulian. Comparative study on mental health status of the elderly under different old-age ways [J]. Chinese Journal of Gerontology, 2003, 23(11): 713 - 715.

[3] Liu Xiaoxi, Chen Yuwen, Bi Kaishun. Learning from the British medical service system to solve the problem of implementing two-way referral system in China [J]. Chinese General Practice, 2013, 16(9): 2926 - 2929.

[4] Chen X, Silverstein M. Intergenerational SocialSupport and the Psychological Well - Being of Older Parents in China [J]. Research on Aging, 2000, 22(1): 43 - 65. 